Published in final edited form as:

Drug Discov Today Dis Mech. 2013 December 1; 10(3-4): e113-e118. doi:10.1016/j.ddmec.2013.03.001.

\title{
STAT3 Signaling in Polycystic Kidney Disease
}

\author{
Thomas Weimbs ${ }^{1}$ and Jeffrey J. Talbot \\ Department of Molecular, Cellular, and Developmental Biology; and Neuroscience Research \\ Institute, University of California, Santa Barbara, CA 93106-9610, U.S.A.
}

\begin{abstract}
Mutations in the gene coding for the integral membrane protein polycystin-1 (PC1) are the cause of most cases of autosomal-dominant polycystic kidney disease (ADPKD), a very common disease that leads to kidney failure and currently lacks approved treatment. Recent work has revealed that PC1 can regulate the transcription factor STAT3, and that STAT3 is aberrantly activated in the kidneys of ADPKD patients and PKD mouse models. Recent approaches to directly inhibit STAT3 in PKD mouse models have been promising. Numerous signaling pathways are known to activate STAT3 and many have long been implicated in the pathogenesis of PKD - such as EGF/EGFR, HGF/c-Met, Src. However, a role of STAT3 in the pathogenesis of PKD had never been considered until now. Here, we review the current findings that suggest that STAT3 is a promising target for the treatment of PKD.
\end{abstract}

\section{Polycystic Kidney Disease}

ADPKD is a very common life-threatening, monogenic disease that is characterized by excessive proliferation and the growth of epithelial-lined cysts that eventually destroy the normal renal parenchyma [1,2]. Most patients eventually progress to renal failure and will require dialysis or kidney transplantation. No approved treatment is currently available to halt or slow disease progression. However, a recent phase 3 trial using a vasopressin $\mathrm{V}_{2^{-}}$ receptor antagonist has shown promise in slowing the decline in kidney function [3]. ADPKD is caused by mutations in the PKD1 or PKD2 genes which encode the proteins polycystin-1 (PC1) and polycystin-2 (PC2), respectively. PC2 is a calcium channel of the TRP family, and forms a complex with PC1. In addition, PC1 - which is mutated in most cases of ADPKD - has been shown to interact with a wide variety of signaling proteins and regulates numerous signaling pathways including heterotrimeric G proteins, wnt-, integrinand JAK/STAT-signaling, and the mTOR pathway. It has remained unclear which of these

\footnotetext{
${ }^{1}$ Corresponding author: Thomas Weimbs, PhD, Department of Molecular, Cellular \& Developmental Biology, And Neuroscience Research Institute, University of California Santa Barbara, Santa Barbara, California 93106-9610, Phone: (805) 893-4144 Fax: (805) 893-4724, weimbs@lifesci.ucsb.edu.

Publisher's Disclaimer: This is a PDF file of an unedited manuscript that has been accepted for publication. As a service to our customers we are providing this early version of the manuscript. The manuscript will undergo copyediting, typesetting, and review of the resulting proof before it is published in its final citable form. Please note that during the production process errors may be discovered which could affect the content, and all legal disclaimers that apply to the journal pertain.

Conflict of interest

The authors have no conflict of interest to declare.
} 
numerous proposed functions is most relevant for understanding the molecular mechanism that leads to renal cyst growth in ADPKD.

The polycystins localize to primary cilia - among other locations - and are required for the function of cilia as sensors of fluid flow on renal epithelial cells. Since mutations in numerous other cilia-associated proteins lead to renal cyst growth, it is currently believed that disruption of the function of primary cilia leads to aberrant proliferation of renal tubule epithelial cells [4] [5]. However, it is unclear what the purpose of this regulation is or which molecular mechanisms are involved.

There are numerous similarities in signaling pathways that are activated both in PKD and in response to kidney injury. This has led to the hypothesis that PKD is a manifestation of aberrant and chronic activation of injury repair pathways that are normally dormant in the healthy kidney but can be rapidly activated in response to insults [6]. Indeed, different forms of renal injury have been shown to trigger rapid renal cyst growth in experimental animal models [7].

Numerous signaling molecules and pathways have been shown to be aberrantly activated in cyst-lining cells in PKD such as Src, Erk and mTOR. Inhibition of many of these pathways leads to significant reductions in renal cyst growth in rodent models of PKD but this has not yet translated into clinical treatments. A case in point are mTOR inhibitors that proved highly effective at high doses in rodent models but were disappointing in subsequent clinical trials [8]. Recent results from several investigators have indicated that STAT3 is aberrantly activated in PKD, that PC1 can regulate STAT3, and that that STAT3 may be a promising drug target for therapy.

\section{STAT3}

Signal Transducer and Activator of Transcription 3 (STAT3) is a member of a protein family composed of seven members (STAT1, 2, 3, 4, 5a, 5b, 6) [9]. Canonical activation of STAT proteins occurs via phosphorylation of a single tyrosine residue within the transactivation domain conserved across the family. This causes homo- or hetero-dimerization and direct translocation to the nucleus where STATs bind specific DNA sequences in complex with transcriptional cofactors to activate gene expression [10]. The cofactors can provide additional gene specificity. STATs can be activated by binding to phospho-tyrosine residues on the cytoplasmic tails of activated cytokine or growth factor receptors (such as IL6 family). This is followed by STAT-phosphorylation via receptor-associated tyrosine kinases of the JAK family, receptor tyrosine kinases (such as EGFR and c-Met), or by nonreceptor tyrosine kinases such as Src [10].

STAT3 is one of the first STATs detectable in embryonic development [11], and loss of STAT3 causes early embryonic lethality [12]. STAT3 confers resistance to apoptosis in many cell types and is considered an oncogene $[13,14]$. It is constitutively activated in many human cancers, and its inhibition leads to inhibition of tumor growth [15] [16] [17] [18] [19, 20]. Intensive efforts are underway to identify STAT3-inhibitory compounds for the development of cancer therapies, but none are yet in clinical use [21] [15, 22] [23]. 


\section{What is the normal role of STAT3 in the kidney?}

Due to its early embryonic lethality, STAT3-null mice have been uninformative for a possible role of STAT3 in renal development. We reported that STAT3 is highly active in renal tubule epithelial cells of mice at postnatal day 7 (d7) when kidneys are actively growing. By d14 STAT3 activity is strongly down-regulated, and almost completely undetectable in adult kidneys [24]. This time-course of STAT3 inactivation coincides with a developmental switch that occurs in the mouse kidney around d14 and leads to cessation of proliferation and kidney growth [25]. In an in vitro culture model with MDCK renal epithelial cells, STAT3 has been shown to be required for hepatocyte growth factor (HGF) induced tubulogenesis $[26,27]$. These studies suggest that STAT3 activity may normally be involved in the regulation of tubule cell proliferation and morphogenesis during renal development.

The fact that STAT3 activity is extremely low in the adult kidney despite high STAT3 expression levels [24] suggests that signaling pathways upstream of STAT3 are not active in the healthy adult kidney and/or that STAT3 activity is strongly suppressed. However, the abundance of STAT3 also suggests that it is ready to be activated at a moment's notice. Indeed, STAT3 was found to be rapidly activated in renal tubule cells in response to numerous forms of insults. $\mathrm{HgCl}_{2}$-induced acute kidney injury in mice causes STAT3 activation that is due - at least in part - to IL6 trans-signaling, a mechanism that involves IL6 and the shed, soluble form of the IL6-receptor [28]. These authors also demonstrated that experimental activation of STAT3 prior to $\mathrm{HgCl}_{2}$ administration dramatically protected animals from AKI and resulted in complete survival. This effect was suggested to involve the induction of reno-protective proteins such as heme oxygenase HO-1 [28]. Similarly, in cultured proximal tubule cells, STAT3 is activated in response to ATP-depletion as a model of renal ischemic injury [29]. Overexpression of constitutively active STAT3 led to increased protection from apoptosis in this system [29]. Renal ischemia reperfusion injury causes increased expression of unphosphorylated STAT3, and strong STAT3 activation by tyrosine-phosphorylation but the affected cell types have not been defined [30] [31] [32, 33].

Unilateral ureteral obstruction (UUO), as a model of obstructive nephropathy, was shown to lead to STAT3 activation. One study found that STAT3 is activated both in tubule epithelial cells and interstitial cells [34] whereas another study reported predominantly activation in interstitial fibroblasts [35]. Treatment with the STAT3 inhibitor S3I-201 led to inhibition of fibrosis and inflammatory cell infiltration [35]. Renal STAT3 activation has also been found in response to adriamycin-induced nephropathy as a model of chronic renal disease but the activated cell types have not been defined [36]. Treatment with the JAK2 inhibitor AG490 was shown to inhibit STAT3 and suppress the long-term renal deterioration in this model [36]

As an overall conclusion, a model emerges in which STAT3 is rapidly activated in response to several forms of renal insults. STAT3 activity appears to be critical for orchestrating the appropriate responses to such insults such as protection from oxidative stress, recruitment of immune cells and tissue regeneration. However, prolonged renal STAT3 activation appears to play a role in destructive processes such as persistent inflammation and fibrosis.

Drug Discov Today Dis Mech. Author manuscript; available in PMC 2015 October 28. 


\section{STAT regulation by PC1}

The initial observation that PC1 can regulate STAT activity was made by Greg Germino's laboratory [37]. These authors showed that overexpression of PC1 causes activation of STAT1 leading to STAT1-mediated upregulation of $\mathrm{p} 21^{\text {waf1 }}$ transcription which induces cell cycle arrest in G0/G1. PC1 was found to bind to JAK2 suggesting that PC1-mediated regulation of JAK2 activity is responsible for STAT1 activation. Finally, PC1-null mouse embryos at E15.5 almost completely lacked tyrosine-phosphorylated STAT1 and expression of $\mathrm{p} 21^{\text {waf } 1}$ suggesting that $\mathrm{PC} 1$ is the master regulator of STAT1/p2 $1^{\text {waf1 }}$ signaling at this developmental stage. STAT3 was also found to be activated by PC1 overexpression although to a lesser degree, and was not further investigated.

Subsequently, our laboratory discovered that PC1 can also regulate STAT6 activity although the mechanism of regulation differed markedly from the regulation of STAT1. We found that the C-terminal cytoplasmic tail of the integral membrane protein $\mathrm{PC} 1$ is released from the membrane by proteolytic cleavage resulting in C-terminal fragments that undergo nuclear translocation, interact with STAT6 and the transcriptional co-activator P100, and coactivate STAT6-dependent gene expression [38]. In contrast, membrane-anchored PC1 inhibited STAT6 activity [38]. STAT6 itself was found to translocate between primary cilia and the nucleus depending on apical fluid flow [38]. Together with the discovery that cleavage of the PC1 tail is regulated by fluid flow [39] these results suggested that PC1mediated regulation of STAT6 activity plays a role in sensing changes of luminal fluid flow and affecting corresponding changes in gene expression [38]. Subsequently, we demonstrated that STAT6 is aberrantly activated in cyst-lining epithelial cells, is part of a positive feedback loop with interleukin 13 and the IL13 receptor, and that inhibition of STAT6 leads to inhibition of renal cyst growth in a PKD mouse model [40]. An important mechanistic distinction to STAT1 is that membrane-anchored PC1 was not able to "activate" STAT6 by tyrosine-phosphorylation but that instead the soluble, cleaved PC1 tail was able to "co-activate" STAT6 that had previously been "activated" by IL13 cytokine signaling.

To clarify the mechanism of STAT regulation by PC1 we re-investigated the effect on STAT1/3. Although we were unable to detect activation of STAT1 by PC1, we discovered a remarkable dual mechanism of the regulation of STAT3 [24]. Membrane-anchored PC1 indeed caused JAK2-dependent activation of STAT3 by tyrosine-phosphorylation, and the membrane-proximal part of the cytoplasmic tail of PC1 was identified as the JAK2 binding site. In addition, however, the cleaved, nuclear PC1 tail was also able to co-activate both STAT3 or STAT1 that had been tyrosine-phosphorylated by cytokine signaling. This indicated that PC1 can regulate STATs at two levels: First, membrane-anchored, full-length PC1 can act similar to an activated growth factor receptor and activate STAT1 and STAT3 by JAK2-mediated tyrosine-phosphorylation. Second, after PC1 is cleaved - e.g. during renal injury - its cytoplasmic tail can co-activate either STAT1, STAT3 or STAT6 depending on which of these STATs has previously been activated by specific growth factors. Therefore, cleaved PC1 cannot by itself activate STAT signaling but it can amplify STAT signaling in response to the growth factor environment of the cell which can lead to different biological responses including proliferation and apoptosis [24]. 


\section{The role of STAT3 in renal cyst growth}

We reported that STAT3 is very strongly activated by tyrosine-phosphorylation in cystlining cells in human ADPKD kidneys and four different PKD mouse models [24]. Independently, two other laboratories also reported strong STAT3 activation in two independent Pkd1 mouse models [41, 42]. Importantly, attempts to inhibit STAT3 in PKD mouse models have led to promising results. Leonard et al. treated Pkd1 mice with high doses of the natural compound curcumin and observed inhibition of renal cyst growth [41]. Curcumin has an extremely broad spectrum of molecular targets including Ser/Thr-kinases (incl. mTOR), Tyr-kinases, growth factor and cytokine receptors, inflammatory enzymes, and several transcription factors including STAT3 [43]. It is possible that the beneficial effect of curcumin observed in Pkd1 mice may be partially due to inhibition of STAT3. Curcumin was also recently found to inhibit cyst growth in an in vitro cell culture system and in embryonic kidney culture but any possible role of STAT3 was not investigated [44]. Another group identified the anti-parasitic compound pyrimethamine as a novel STAT3 inhibitor and showed that it inhibits renal STAT3 activity and renal cyst growth in a Pkd1 mouse model [42]. Similar results were obtained using another STAT3 inhibitor, S3I-201 [42]. Even though the specificity towards STAT3 of these compounds is either poor or not well established, altogether these studies suggest that STAT3 may be a highly promising therapeutic target for treatment of PKD. More specific inhibitors or genetic approaches are needed to define the contribution of STAT3 as a driver of renal cyst growth.

What are the upstream activators of STAT3 in PKD? Since PC1, the protein affected in most cases of ADPKD, regulates STAT3 [37] [24] it is reasonable to assume that PC1 may play a role in the aberrant activation of STAT3 in renal cysts. However, the picture appears to be rather complicated. Two pathogenic patient mutations were identified that altered the ability of membrane-anchored PC1 to activate STAT3, however, one mutation increased STAT3 activation while another mutation diminished it [24]. Furthermore, renal cyst growth can be caused by both reducing/eliminating expression of PC1 (e.g. in conditional KO models or by hypomorphic alleles) and by overexpression of PC1 [45]. In kidneys of ADPKD patients, PC1 has consistently been found to be overexpressed rather than being absent. Indeed, the cleaved, C-terminal tail of PC1 is strongly overexpressed in kidneys from ADPKD patients [24] and a PKD mouse model [39]. Since the PC1 tail has the ability to co-activate STAT signaling in response to cytokine/growth factor activity [24] it is possible that the observed STAT3 activation in PKD is due to a combination of STAT3-activating cytokines and the signal-amplifying property of the PC1 tail.

Several growth factors and upstream activators of STAT3 have been implicated in PKD including epidermal growth factor (EGF) and its receptor (EGFR), HGF and its receptor cMet, and Src. EGF [46] and HGF [47] are both elevated in PKD kidneys and found in cyst fluid, the EGFR is overexpressed and mis-targeted to the apical plasma membrane in cystlining cells [46], and overexpression of c-Met leads to polycystic kidneys [48]. Treatment of PKD mouse models with EGFR inhibitors [49] and treatment of Pkd1-null embryos with a c-Met inhibitor [50] reduce renal cyst growth. A possible link between PC1 and c-Met/ EGFR signaling has been uncovered when it was found that the loss of PC1 leads to a trafficking defect of the E3-ubiquitin ligase $\mathrm{c}-\mathrm{Cbl}$ which is required for the downregulation 
of MET and EGFR after receptor activation [50]. Furthermore, Src - a tyrosine kinase that can activate STAT3 directly - is aberrantly activated in PKD, and the Src inhibitor SKI-606 reduces renal cyst growth in PKD mice [51]. The immune system may also play a likely role in secreting STAT3-activating cytokines in PKD. For example, IL6 is secreted by T-cells and macrophages, and IL6-trans-signaling has been shown to activate STAT3 in renal tubule cells in response to AKI [28]. Macrophages were recently shown to promote cyst growth in PKD [52]. Interestingly, cystic epithelial cells secrete macrophage chemoattractants including MCP-1 [52] whose expression is known to be driven by STAT3 [53]. An interesting speculation is that STAT3-dependent expression of macrophage chemoattractants by tubule epithelial cells leads to macrophage recruitment which, in turn, further activate STAT3 in these cells by cytokine signaling. Such an interplay between renal epithelial cells and immune cells could lead to a vicious cycle of mutual positive feedback stimulation that causes persistent STAT3 activation and eventually cyst growth and fibrosis.

In addition to activation by tyrosine-phosphorylation, STAT3 is regulated by Ser727phosphorylation by mTOR- and ERK-dependent pathways [54] which are known to be activated in PKD [55, 56]. However, the situation is complicated by the fact that Ser727 phosphorylation can lead both to increased and decreased nuclear STAT3 activity [57] [58]. Unphosphorylated STAT3 (U-STAT3) can also regulate gene expression which leads to a more sustained effect than the canonical effects of Tyr-phosphorylated STAT3 [59]. Increased expression of U-STAT3 has been observed in PKD mouse models [24, 41, 42]. USTAT3 has been suggested to play a role in increased expression of pro-fibrotic/ inflammatory genes in acute kidney injury [30]. U-STAT3 can also increase the expression of c-Met [60] which could potentially be involved in the observed upregulation of c-Metsignaling in PKD.

Given the abundance of over-activated pathways that signal via STAT3 it is almost surprising that the role of STAT3 in PKD has only very recently been investigated. It is currently unknown which of the biological effects of STAT3-dependent gene activation may be most relevant in the pathogenesis of PKD. Based on the known roles of STAT3 in promoting proliferation, survival and resistance to apoptosis in cancer cells, these are obvious candidates. But other effects should not be discounted. For example, STAT3 drives the expression of heme oxygenase (HO) in response to kidney injury which is thought to lead to protection from oxidative stress and increased cell survival [28]. A role of HO activity in the regulation of renal cyst growth has recently been identified [61]. Furthermore, STAT3 is required for HIF-1a RNA expression under both hypoxia and growth signaling conditions (Niu, 2008). HIF-1a is an important regulator of tumor growth and angiogenesis and has been found to be up-regulated in the renal cyst-lining epithelium and implicated in the progression of PKD (Bernhardt, 2007).

\section{Conclusions}

Independent work from several laboratories has clearly established that STAT3 is strongly activated by tyrosine-phosphorylation in human ADPKD and several mouse models. Numerous STAT3-activating signaling pathways have already been shown to be involved in renal cyst growth in PKD. Initial approaches at directly inhibiting STAT3 activity in PKD 
mouse models have been promising and it appears very likely that STAT3 is a valid target for PKD therapy. However, more specific experimental approaches are needed to clearly define the role of STAT3 in the pathogenesis of PKD. Another important challenge is to define the purpose of the regulation of STAT3 activity by PC1 both in the normal kidney and under pathological conditions. STAT3 is a hotly pursued target for the treatment of numerous types of cancer and it is likely that clinically useful drugs will emerge in the future. Such compounds should be tested for their potential efficacy for the treatment of PKD.

\section{Acknowledgments}

Supported by a National Institutes of Health grant (DK62338) and a Santa Barbara Cottage Hospital - UCSB, Special Research Award for Biomedical Science to T.W.

\section{Bibliography}

1. Harris PC, Torres VE. Polycystic kidney disease. Annu Rev Med. 2009; 60:321-337. [PubMed: 18947299]

2. Gallagher AR, et al. Molecular advances in autosomal dominant polycystic kidney disease. Adv Chronic Kidney Dis. 2010; 17(2):118-130. [PubMed: 20219615]

3. Torres VE, et al. Tolvaptan in Patients with Autosomal Dominant Polycystic Kidney Disease. N Engl J Med. 2012

4. Zhou J. Polycystins and primary cilia: primers for cell cycle progression. Annu Rev Physiol. 2009; 71:83-113. [PubMed: 19572811]

5. Berbari NF, et al. The primary cilium as a complex signaling center. Curr Biol. 2009; 19(13):R526R535. [PubMed: 19602418]

6. Weimbs T. Polycystic kidney disease and renal injury repair: common pathways, fluid flow, and the function of polycystin-1. Am J Physiol Renal Physiol. 2007; 293(5):F1423-F1432. [PubMed: 17715262]

7. Weimbs T. Third-hit signaling in renal cyst formation. J Am Soc Nephrol. 2011; 22(5):793-795. [PubMed: 21493772]

8. Watnick T, Germino GG. mTOR inhibitors in polycystic kidney disease. N Engl J Med. 2010; 363(9):879-881. [PubMed: 20581393]

9. Stark GR, Darnell JE Jr. The JAK-STAT pathway at twenty. Immunity. 2012; 36(4):503-514. [PubMed: 22520844]

10. Levy DE, Darnell JE Jr. Stats: transcriptional control and biological impact. Nat Rev Mol Cell Biol. 2002; 3(9):651-662. [PubMed: 12209125]

11. Oates AC, et al. Zebrafish stat 3 is expressed in restricted tissues during embryogenesis and stat 1 rescues cytokine signaling in a STAT1-deficient human cell line. Dev Dyn. 1999; 215(4):352-370. [PubMed: 10417824]

12. Takeda K, et al. Targeted disruption of the mouse Stat 3 gene leads to early embryonic lethality. Proc Natl Acad Sci U S A. 1997; 94(8):3801-3804. [PubMed: 9108058]

13. Stepkowski SM, et al. STAT3: an important regulator of multiple cytokine functions. Transplantation. 2008; 85(10):1372-1377. [PubMed: 18497672]

14. Avalle L, et al. STAT1 and STAT3 in tumorigenesis: A matter of balance. JAKSTAT. 2012; 1(2): 65-72. [PubMed: 24058752]

15. Debnath B, et al. Small Molecule Inhibitors of Signal Transducer and Activator of Transcription 3 (Stat3) Protein. J Med Chem. 2012

16. Schlessinger K, Levy DE. Malignant transformation but not normal cell growth depends on signal transducer and activator of transcription 3. Cancer Res. 2005; 65(13):5828-5834. [PubMed: 15994959] 
17. Lin L, et al. Novel STAT3 phosphorylation inhibitors exhibit potent growth-suppressive activity in pancreatic and breast cancer cells. Cancer Res. 2010; 70(6):2445-2454. [PubMed: 20215512]

18. Onimoe GI, et al. Small molecules, LLL12 and FLLL32, inhibit STAT3 and exhibit potent growth suppressive activity in osteosarcoma cells and tumor growth in mice. Invest New Drugs. 2011

19. Siddiquee K, et al. Selective chemical probe inhibitor of Stat3, identified through structure-based virtual screening, induces antitumor activity. Proc Natl Acad Sci U S A. 2007; 104(18):73917396. [PubMed: 17463090]

20. Darnell JE. Validating Stat3 in cancer therapy. Nat Med. 2005; 11(6):595-596. [PubMed: 15937466]

21. Zhao M, et al. Small molecule inhibitors of STAT3 for cancer therapy. Curr Med Chem. 2011; 18(26):4012-4018. [PubMed: 21824090]

22. Lavecchia A, et al. STAT-3 inhibitors: state of the art and new horizons for cancer treatment. Curr Med Chem. 2011; 18(16):2359-2375. [PubMed: 21568920]

23. Page BDG, et al. Signal transducer and activator of transcription 3 inhibitors: a patent review. Expert Opin Ther Pat. 2011; 21(1):65-83. [PubMed: 21114420]

24. Talbot JJ, et al. Polycystin-1 regulates STAT activity by a dual mechanism. Proc Natl Acad Sci U S A. 2011; 108(19):7985-7990. [PubMed: 21518865]

25. Piontek K, et al. A critical developmental switch defines the kinetics of kidney cyst formation after loss of Pkd1. Nat Med. 2007; 13(12):1490-1495. [PubMed: 17965720]

26. Boccaccio C, et al. Induction of epithelial tubules by growth factor HGF depends on the STAT pathway. Nature. 1998; 391(6664):285-288. [PubMed: 9440692]

27. Santos OF, et al. Involvement of hepatocyte growth factor in kidney development. Dev Biol. 1994; 163(2):525-529. [PubMed: 8200486]

28. Nechemia-Arbely Y, et al. IL-6/IL-6R axis plays a critical role in acute kidney injury. J Am Soc Nephrol. 2008; 19(6):1106-1115. [PubMed: 18337485]

29. Wang J, et al. STAT3 inhibits apoptosis of human renal tubular epithelial cells induced by ATP depletion/recovery. Nephron Exp Nephrol. 2008; 108(1):e11-e18. [PubMed: 18097150]

30. Arany I, et al. A novel U-STAT3-dependent mechanism mediates the deleterious effects of chronic nicotine exposure on renal injury. Am J Physiol Renal Physiol. 2012; 302(6):F722-F729. [PubMed: 22169004]

31. Arany I, et al. STAT3 attenuates EGFR-mediated ERK activation and cell survival during oxidant stress in mouse proximal tubular cells. Kidney Int. 2006; 70(4):669-674. [PubMed: 16788692]

32. Ogata K, et al. Upregulation of HNF-1beta during experimental acute kidney injury plays a crucial role in renal tubule regeneration. Am J Physiol Renal Physiol. 2012

33. Yang N, et al. Blockage of JAK/STAT signalling attenuates renal ischaemiareperfusion injury in rat. Nephrol Dial Transplant. 2008; 23(1):91-100. [PubMed: 17670769]

34. Kuratsune M, et al. Signal transducer and activator of transcription 3 involvement in the development of renal interstitial fibrosis after unilateral ureteral obstruction. Nephrology (Carlton). 2007; 12(6):565-571. [PubMed: 17995582]

35. Pang M, et al. A novel STAT3 inhibitor, S3I-201, attenuates renal interstitial fibroblast activation and interstitial fibrosis in obstructive nephropathy. Kidney Int. 2010; 78(3):257-268. [PubMed: 20520592]

36. Li R, et al. Inhibition of Jak/STAT signaling ameliorates mice experimental nephrotic syndrome. Am J Nephrol. 2007; 27(6):580-589. [PubMed: 17823504]

37. Bhunia AK, et al. PKD1 induces p21(waf1) and regulation of the cell cycle via direct activation of the JAK-STAT signaling pathway in a process requiring PKD2. Cell. 2002; 109(2):157-168. [PubMed: 12007403]

38. Low SH, et al. Polycystin-1, STAT6, and P100 function in a pathway that transduces ciliary mechanosensation and is activated in polycystic kidney disease. Dev Cell. 2006; 10(1):57-69. [PubMed: 16399078]

39. Chauvet V, et al. Mechanical stimuli induce cleavage and nuclear translocation of the polycystin-1 C terminus. J Clin Invest. 2004; 114(10):1433-1443. [PubMed: 15545994] 
40. Olsan EE, et al. Signal transducer and activator of transcription-6 (STAT6) inhibition suppresses renal cyst growth in polycystic kidney disease. Proc Natl Acad Sci U S A. 2011; 108(44):1806718072. [PubMed: 22025716]

41. Leonhard WN, et al. Curcumin inhibits cystogenesis by simultaneous interference of multiple signaling pathways: in vivo evidence from a Pkd1-deletion model. Am J Physiol Renal Physiol. 2011; 300(5):F1193-F1202. [PubMed: 21345977]

42. Takakura A, et al. Pyrimethamine inhibits adult polycystic kidney disease by modulating STAT signaling pathways. Hum Mol Genet. 2011; 20(21):4143-4154. [PubMed: 21821671]

43. Kunnumakkara AB, et al. Curcumin inhibits proliferation, invasion, angiogenesis and metastasis of different cancers through interaction with multiple cell signaling proteins. Cancer Lett. 2008; 269(2):199-225. [PubMed: 18479807]

44. Gao J, et al. Curcumin inhibits renal cyst formation and enlargement in vitro by regulating intracellular signaling pathways. Eur J Pharmacol. 2011; 654(1):92-99. [PubMed: 21187084]

45. Harris PC. What is the role of somatic mutation in autosomal dominant polycystic kidney disease? J Am Soc Nephrol. 2010; 21(7):1073-1076. [PubMed: 20488953]

46. Sweeney WE Jr, Avner ED. Molecular and cellular pathophysiology of autosomal recessive polycystic kidney disease (ARPKD). Cell Tissue Res. 2006

47. Horie S, et al. Mediation of renal cyst formation by hepatocyte growth factor. Lancet. 1994; 344(8925):789-791. [PubMed: 7916076]

48. Takayama H, et al. Renal tubular hyperplasia, polycystic disease, and glomerulosclerosis in transgenic mice overexpressing hepatocyte growth factor/scatter factor. Lab Invest. 1997; 77(2): 131-138. [PubMed: 9274855]

49. Torres VE, et al. EGF receptor tyrosine kinase inhibition attenuates the development of PKD in Han:SPRD rats. Kidney Int. 2003; 64(5):1573-1579. [PubMed: 14531789]

50. Qin S, et al. Failure to ubiquitinate c-Met leads to hyperactivation of mTOR signaling in a mouse model of autosomal dominant polycystic kidney disease. J Clin Invest. 2010; 120(10):3617-3628. [PubMed: 20852388]

51. Sweeney WE Jr, et al. Src inhibition ameliorates polycystic kidney disease. J Am Soc Nephrol. 2008; 19(7):1331-1341. [PubMed: 18385429]

52. Karihaloo A, et al. Macrophages promote cyst growth in polycystic kidney disease. J Am Soc Nephrol. 2011; 22(10):1809-1814. [PubMed: 21921140]

53. Chatterjee PK, et al. Cholinergic agonists regulate JAK2/STAT3 signaling to suppress endothelial cell activation. Am J Physiol Cell Physiol. 2009; 297(5):C1294-C1306. [PubMed: 19741199]

54. Yokogami K, et al. Serine phosphorylation and maximal activation of STAT3 during CNTF signaling is mediated by the rapamycin target mTOR. Curr Biol. 2000; 10(1):47-50. [PubMed: 10660304]

55. Shillingford JM, et al. The mTOR pathway is regulated by polycystin-1, and its inhibition reverses renal cystogenesis in polycystic kidney disease. Proc Natl Acad Sci U S A. 2006; 103(14):54665471. [PubMed: 16567633]

56. Calvet JP. MEK Inhibition Holds Promise for Polycystic Kidney Disease. J Am Soc Nephrol. 2006; 17(6):1498-1500. [PubMed: 16687624]

57. Chung J, et al. STAT3 serine phosphorylation by ERK-dependent and - independent pathways negatively modulates its tyrosine phosphorylation. Mol Cell Biol. 1997; 17(11):6508-6516. [PubMed: 9343414]

58. Wegrzyn J, et al. Function of mitochondrial Stat3 in cellular respiration. Science. 2009; 323(5915): 793-797. [PubMed: 19131594]

59. Yang J, Stark GR. Roles of unphosphorylated STATs in signaling. Cell Res. 2008; 18(4):443-451. [PubMed: 18364677]

60. Yang J, et al. Novel roles of unphosphorylated STAT3 in oncogenesis and transcriptional regulation. Cancer Res. 2005; 65(3):939-947. [PubMed: 15705894]

61. Zhou J, et al. Kidney Injury Accelerates Cystogenesis via Pathways Modulated by Heme Oxygenase and Complement. J Am Soc Nephrol. 2012 


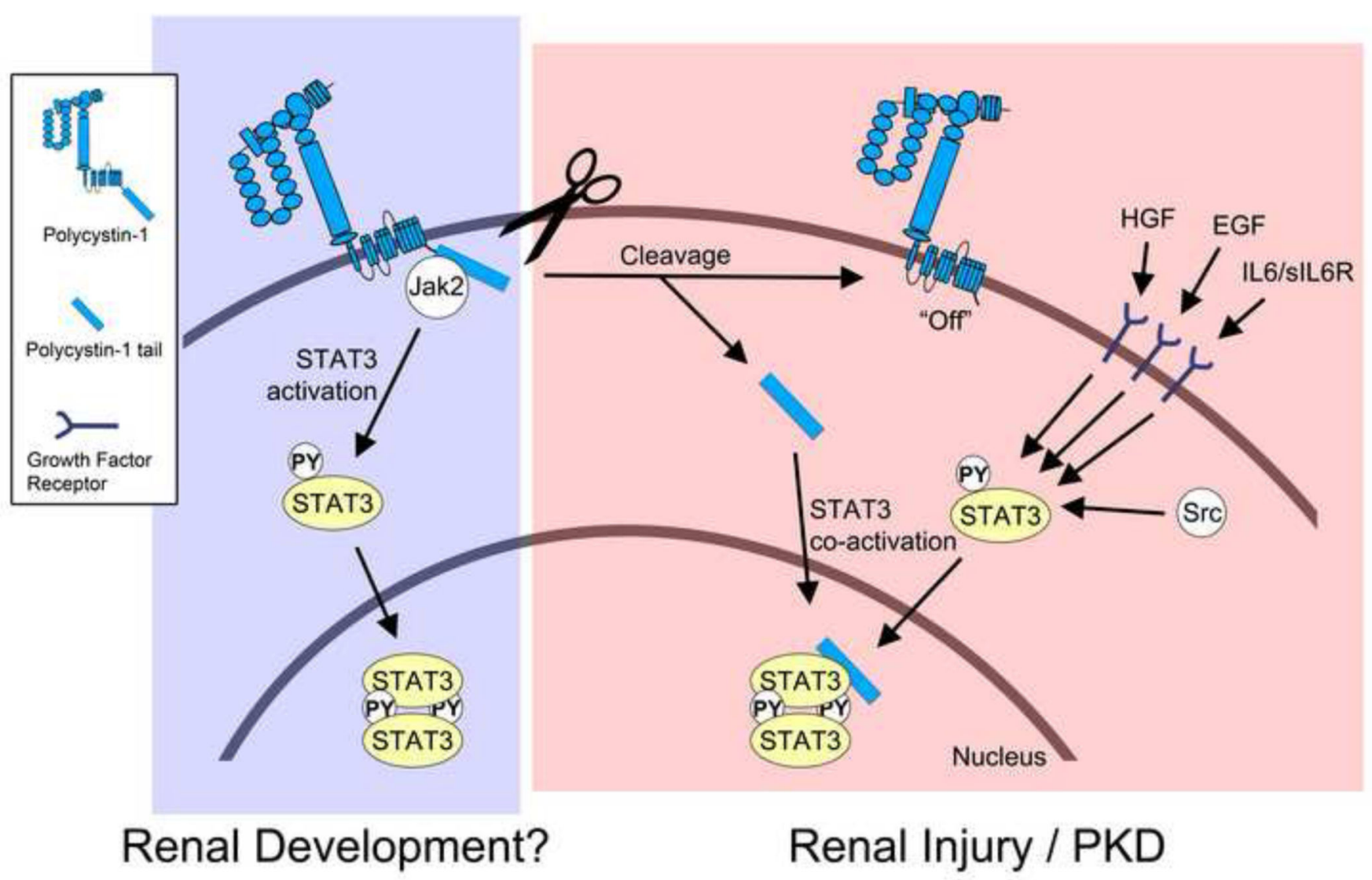

Figure. Schematic of current model of STAT3 signaling in the normal and diseased kidney During renal development, membrane-anchored, full-length PC1 may cause direct activation of STAT3 via JAK2 that is associated with its C-terminal cytoplasmic tail. Direct STAT3 activation by PC1 would be an intrinsic pathway that is independent of growth factormediated STAT3 signaling. It is currently unknown how the activation of STAT3 by fulllength PC1 is regulated. It is possible that an - yet unidentified - extracellular ligand may trigger STAT3 activation, or that the extracellular domain of PC1 engages in homo-typic interactions. It is also possible that fluid flow may regulate this activity. During renal injury and in PKD PC1 appears to undergo proteolytic cleavage that releases its cytoplasmic tail into the cytoplasm. This turns "off" the ability of the remaining membrane-anchored portion of PC1 to activate STAT3. However, the soluble PC1 tail can now translocate to the nucleus and co-activate STAT3 that has been activated by prior growth factor signaling. In addition to STAT3, the cleaved PC1 tail can also co-activate STAT1 and STAT6 (not shown here). Therefore, the cleaved PC1 tail may have the ability to amplify different signaling pathways that lead to different cellular responses depending on the growth factor and cytokine environment. 